\title{
A different protein corona cloaks "true-to-life" nanoplastics with respect to synthetic polystyrene nanobeads
}

\author{
Serena Ducolia,b, Stefania Federici ${ }^{b, c,{ }^{*}}$, Roland Nicsanu ${ }^{d}$, Andrea Zendrinid,e, Claudio Marchesi ${ }^{b, c}$, \\ Lucia Paolini $^{\mathrm{d}, \mathrm{e}}$, Annalisa Radeghierid,e $^{\mathrm{d}}$, Paolo Bergese ${ }^{\mathrm{b}, \mathrm{d}, \mathrm{e}}$ and Laura E. Depero ${ }^{\mathrm{b}, \mathrm{c}}$
}

\author{
a. Department of Information Engineering, University of Brescia, Brescia, Italy \\ b. National Interuniversity Consortium of Materials Science and Technology (INSTM), Florence, Italy \\ c. Department of Mechanical and Industrial Engineering, University of Brescia, Brescia, Italy \\ d. Department of Molecular and Translational Medicine, University of Brescia, Brescia, Italy \\ e. Consorzio Sistemi a Grande Interfase (CSGI), Department of Chemistry, University of Florence, Sesto \\ Fiorentino, Italy \\ *stefania.federici@unibs.it
}

\begin{abstract}
Given the complexity of separating nanoplastics from environmental samples, studies have usually been conducted using synthetic polystyrene nanobeads. By mechanical fragmentation in cryogenic conditions of daily-life plastic items, we produced "true-to-life" nanoplastics (T2LNPs), that promises to give a true insight into the interaction with biological systems. T2LNPs have been fully characterized by Fourier transform Infrared spectroscopy and by Atomic Force Microscopy. They result in populations of spheroidal nanoparticles with a broad multimodal size distribution. The mandatory need for a representative sample to evaluate the potential effects of nanoparticles on human health and the environment is demonstrated by the different protein corona identified on T2LNPs and synthetic polystyrene nanobeads upon incubation with human plasma.
\end{abstract}

\section{Keywords}

Nanoplastics, Nanobeads, Biological systems, Interface, Protein corona.

\section{Synopsis}

"True-to-life" nanoplastics obtained by mechanical fragmentation of polystyrene-based daily-life items are cloaked in human plasma by a different protein corona with respect to synthetic polystyrene nanobeads. 


\section{Introduction}

Environmental plastic pollution is nowadays a great concern. The extremely wide usage of plastic in almost every human activity has led to a progressive accumulation of plastic waste in the environment. ${ }^{1,2}$ Also, the ongoing COVID-19 pandemic is worsening the situation due to the huge consumption of disposable surgical masks and gloves and their incorrect abandonment in the environment. ${ }^{3}$ Once plastic enters the environment, especially oceans and seas, it undergoes abiotic and biotic degradation processes, such as mechanical fragmentation induced by water and marine rocks action, ${ }^{4}$ photodegradation, ${ }^{4}$ and biological metabolism. ${ }^{5}$ Plastic degradation results in progressive changes of physical properties (such as crystallinity and mechanical integrity), ${ }^{6}$ embrittlement, and fragmentation into smaller pieces, generating micro- and nanoscale particles. ${ }^{7}$ The need to understand the possible implications of microplastics and nanoplastics pollution on the environment and living organisms is becoming increasingly pressing.

Even if microplastics have been largely documented in nature, ${ }^{8}$ often associated with organic and inorganic pollutants ${ }^{9,10,11}$ and demonstrating severe damages to living organisms, ${ }^{12,13,14}$ different considerations must be accounted for nanoplastics. Literature regarding nanoplastics is growing exponentially, but due to the complexity of separating nanoplastics from environmental samples, ${ }^{15,16}$ most of the studies conducted so far have been carried out on model nanoparticles, such as synthetic polystyrene nanobeads. ${ }^{17,18,19,20}$ The toxicity of these synthetic polymeric nanoparticles has been extensively investigated on cells and marine organisms at different trophic levels, evidencing several biological dysfunctions. . $^{21,22,23,24,25,26,10}$ In addition, nanoplastics could be even more dangerous than their micrometric counterpart because, due to nanometric size, they are thought to be able to penetrate tissues and biological barriers. ${ }^{27,28}$

However, despite this bulk of information, several knowledge gaps still exist about the behavior and characteristics of naturally-occurring nanoplastics. Nanobeads synthesized in the framework of colloidal chemistry, following bottom-up approaches, share no analogies with nanoplastics forming in nature, following top-down degradation pathways. ${ }^{17}$ Pristine nanoparticles usually are uniform in size, shape, composition, while naturally-occurring nanoplastics are expected to be highly heterogeneous in size, have irregular shapes, and complex surface chemistry (Figure 1). ${ }^{29}$ There is an urgent need to create nanomaterials that better reflect the real characteristics of nanoplastics naturally formed, viz. true-to-life nanoplastics (T2LNPs), to close the gap between the laboratory parameters and the rules of nature, and to provide more realistic understandings of the characteristics of nanoplastics. To date, some attempts have been made to generate micro- and nanoplastics by fragmentation procedures, following different top-down approaches mimicking the natural processes of environmental degradation. ${ }^{29,30,31,32,33}$ These approaches cannot account for all the simultaneous mechanisms that contribute to the degradation of plastic in nature, but represent a step forward in closing the gap in the nanotoxicology of environmental samples.

Nevertheless, interactions of fragmentally generated nanoplastics and biological systems are far from being understood. When a nanoparticle gets in contact with a complex biological environment, its surface is 
immediately covered by different biomolecules that form a biological corona. ${ }^{34}$ Currently, the protein component of this interaction is the most studied in nanoplastics toxicology. ${ }^{35,36,37,38,39}$ The hard protein corona, hereinafter referred as protein corona, provides a new identity to nanoparticles and determines their interactions and effects on cells, tissues, and organisms. ${ }^{34}$ Literature about protein corona on nanobeads is extensive and well documented, but there is no reason for environmental nanoplastics to share the same behaviour.

In this paper, we present a study on T2LNPs production and characterization and the protein corona formation with respect to synthetic standard nanobeads (nanobeads). First, we investigated the nanoplastics formation from daily life plastic items subjected to a mechanical fragmentation through an ultracentrifugal mill operating in cryogenic conditions. The produced nanoplastics were characterized by Fourier transform Infrared (FT-IR) spectroscopy to investigate their chemical nature and check the absence of induced chemical modifications. Morphology and size distribution analyses were performed through Atomic Force Microscope (AFM), giving exciting insights compared to nanobeads. Finally, the protein corona formation from human plasma on T2LNPs and nanobeads examined, highlighting differences in protein corona compositions.
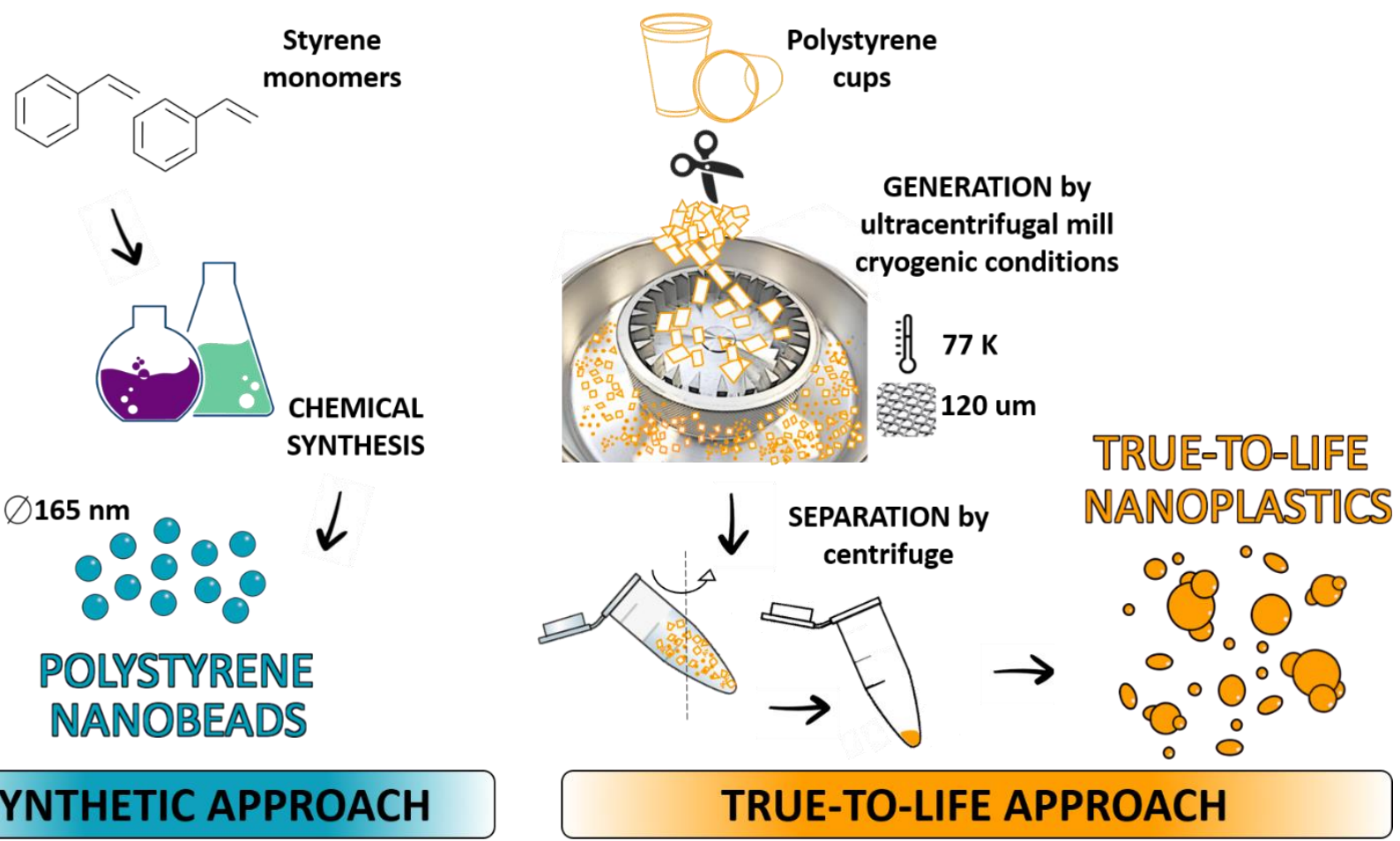

Figure 1. The synthetic and the true-to-life approaches for generating nanoplastics for controlled experiments. 


\section{Materials and methods}

\section{Materials}

Commonly used polystyrene-based disposable items (coffee cups) were used to produce true-to-life nanoplastics (T2LNPs) samples by means of mechanical fragmentation. Synthetic polystyrene nanobeads, hereafter referred as nanobeads, with a nominal diameter of $165 \mathrm{~nm}$ were purchased from microParticles $\mathrm{GmbH}$. Nanobeads were diluted at $50 \mu \mathrm{g} / \mathrm{ml}$ with MilliQ-water immediately before use and characterized by FT-IR spectroscopy and AFM.

\section{Preparation of T2LNPs samples}

Polystyrene-based disposable items were manually cut with steel scissors, washed with Milli-Q water together with a few drops of commercial detergent and externally sonicated in a water bath for 15 minutes in order to wash the pieces and remove dirt and grease. Three washing cycles were then performed with Milli-Q water, and then fragments were dried in air at room temperature. Polystyrene pieces were embrittled in liquid nitrogen for 30 minutes, then transferred into an ultracentrifugal mill (ZM 200, Retsch) previously cooled down with liquid nitrogen. Pieces were blended at 18,000 rpm $(17,963 \mathrm{~g})$ until complete fragmentation (about 60 seconds) under a continuous flow of liquid nitrogen in order to avoid overheating of the rotor with consequent degradation of the polymer. The mill was equipped with a $120 \mu \mathrm{m}$ mesh sieve, acting as a first fractionation step for nanoplastics. The powder was collected and stored in a falcon tube.

\section{Pre-treatment of Milli-Q water}

All experiments were conducted in centrifuged Milli-Q water. The preparation of Milli-Q water is a crucial step to avoid unknown contamination in the final concentrated pellet of T2LNPs. Milli-Q water was carefully pre-treated before the use by centrifugation at 16,000 g for 45 minutes (Avanti J25, rotor ja20, polycarbonate tubes 357003 Beckman) to separate all the interfering suspended particles. Supernatant was collected leaving $1 \mathrm{~cm}$ of Milli-Q water not to disturb pelleted material.

\section{Separation and concentration of T2LNPS}

About $0.4 \mathrm{~g}$ of powder originated from the fragmentation procedure was suspended in pre-treated Milli-Q water and externally sonicated in a water bath for about 30 minutes. To recover and concentrate T2LNPs from the medium, a protocol of centrifugations was applied. Samples were subjected to two sequential centrifuge steps. The first step was conducted at $500 \mathrm{~g}$ for 45 minutes (5804R Eppendorf centrifuge, A-4-44 rotor, $50 \mathrm{~mL} 174 \times 22 \mathrm{~mm}$ polypropylene tube Sarstedt). Supernatants were transferred into new centrifuge tubes and then centrifuged at 16,000 g for 45 minutes (Avanti J25, rotor ja20, polycarbonate tubes 357003 Beckman); pellets were preserved while supernatants were discarded.

\section{T2LNPs characterization by FT-IR spectroscopy}

FT-IR measurements were performed with an Equinox 55 spectrometer (Bruker) operating in transmission mode. Pellets of T2LNPs were resuspended in Milli-Q water and briefly sonicated in a water bath for 5 minutes to promote nanoparticles resuspension. From each sample a $2 \mu \mathrm{L}$ droplet was deposited onto a 
diamond window and dried under a mild stream of nitrogen. Measurements were performed at a nominal resolution of $4 \mathrm{~cm}^{-1}$ in the range of $4000-400 \mathrm{~cm}^{-1}$. To increase the signal-to-noise ratio, 128 scans per samples were co-added without change the position of the sample between each scan (time per scan 0.9 seconds, included dead time). For all spectra manipulations OPUS software 5.0 was used.

\section{T2LNPs morphological characterization with AFM}

Pellets of T2LNPs were resuspended in Milli-Q water and briefly sonicated in a water bath for 5 minutes to promote nanoparticles resuspension. Three $\mu \mathrm{l}$ of the samples were spotted on a freshly cleaved roundshaped mica sheet (Grade V-1, thickness $0.1 \mathrm{~mm}$, diameter $10 \mathrm{~mm}$ ) and air-dried over a heating plate at 37$40^{\circ} \mathrm{C}$ (Velp Scientifica). Dried samples were then imaged in tapping mode with JSPM-4210 AFM microscope (JEOL) equipped with NSC35/ALBS (MikroMasch) ultrasharp tips (Resonant Frequency $\approx 205 \mathrm{kHz}$; Force Constant $\approx 8.9 \mathrm{~N} / \mathrm{m}$, typical radius tip $<10 \mathrm{~nm}$ ). Topography images were collected over different length scales (scan size ranged from $2 \mu \mathrm{m}$ to $25 \mu \mathrm{m}$ with a scan speed of ranged from $9.23 \mu \mathrm{m} / \mathrm{s}$ to $46.2 \mu \mathrm{m} / \mathrm{s}$ ). The obtained topography images were processed with WSxM 5.0 software ${ }^{40}$ and Gwyddion software. The same AFM protocol was also applied for the AFM images of T2LNPs after the formation of the protein corona.

\section{Size distribution analysis of T2LNPs samples}

Size distribution analysis was performed on AFM images. Briefly, images of different length scale (from 2 $\mu \mathrm{m}$ to $25 \mu \mathrm{m}$ ) were obtained by AFM as previously described; images of different length scale were necessary to cover the extremely high polydispersity of the T2LNPs samples and considered both single particles and big aggregates. Images were processed using Gwyddion software. About 20,000 items were counted for size distribution and both the z-dimension (height) and the diameter of particles were selected for the analysis in order to avoid tip-driven AFM artefacts and to account also for in-plane T2LNPs aggregates. Two different independent T2LNPs samples were analyzed, and data collected for the final distributions.

\section{Human blood collection and plasma preparation}

Peripheral blood was obtained from healthy subjects (Geriatrics Unit, Civil Hospitals of Brescia, Italy). The study was approved by the ethics committee of Civil Hospitals of Brescia on 8th June 2016 (Informed consent was obtained from all the subjects enrolled in the study). EDTA was used as the anticoagulant. Blood samples were processed within $2 \mathrm{~h}$ from the withdrawal and kept at room temperature. Plasma-EDTA (plasma) was centrifuged at $800 \mathrm{~g}$ for $10 \mathrm{~min}$ (5804R Eppendorf Centrifuge, A-4-44 rotor, 15 $\mathrm{ml}$ tubes), 2,500 g for $15 \mathrm{~min}$ and then centrifuged a second time at 2,500 $\mathrm{g}$ for $15 \mathrm{~min}$. All centrifugation steps were made at room temperature with low acceleration and avoiding the application of the centrifuge brake. After each centrifugation plasma was collected in a fresh plastic tube, leaving $1 \mathrm{~cm}$ of plasma above the buffy layer to not disturb it. Plasma was finally transferred into cryo-vials in $1 \mathrm{ml}$ aliquots and stored at $-80^{\circ} \mathrm{C} .{ }^{41}$ 


\section{Protein corona formation}

Pellets of T2LNPs were resuspended in Milli-Q water, briefly sonicated in a water bath for 5 minutes to promote nanoparticles resuspension, and incubated with plasma diluted $1: 2$ in Milli-Q water for $1 \mathrm{~h}$ at $4^{\circ} \mathrm{C}$. After incubation, samples were diluted 1:2 with Milli-Q water and centrifuged at 16,000 g for 45 minutes at $4^{\circ} \mathrm{C}$ (5417C Eppendorf centrifuge, 45-30-11 rotor) to pellet the nanoparticle-protein complexes and separate from the supernatant plasma. Pellets were then subjected to three washing steps by resuspending in $500 \mu$ of Milli-Q water and centrifuging at $16,000 \mathrm{~g}$ for 45 minutes at $4^{\circ} \mathrm{C}$. Pellets were then stored at $4^{\circ} \mathrm{C}$ for AFM, FT-IR, and SDS-PAGE experiments.

The same protocol was also applied to nanobeads samples with a concentration of $50 \mu \mathrm{g} / \mathrm{ml}$.

\section{SDS-PAGE of protein corona samples}

After the third washing step, pellets were resuspended in $20 \mu \mathrm{l}$ of Milli-Q water and briefly sonicated in a water bath for 5 minutes. Four $\mu \mathrm{l}$ of reducing sample buffer $6 \mathrm{X}$ (480mM Tris-HCl pH 6.8; $12 \%$ SDS; $45 \%$ Glycerin; 0,06\% Bromophenol blue, 12\% -mercaptoethanol) were added, and the samples were boiled at $95^{\circ} \mathrm{C}$ for 5 minutes. Samples were loaded in $7.5 \%$ polyacrylamide gel (30\% acrylamide and bis-acrylamide solution, 29:1 Bio-Rad). Gel electrophoresis was performed at $140 \mathrm{~V}$, for about $60 \mathrm{~min}$. Gels were stained with Imperial protein stain (Thermo-Fisher Scientific) for $2 \mathrm{~h}$ at room temperature and then destained in Milli-Q water overnight at room temperature. The absence of non-specific signals arising from bare T2LNPs and nanobeads was also checked by SDS-PAGE analysis (results reported in Figure S6 in Supporting Information). Images were acquired using a G:Box Chemi XT Imaging system. ${ }^{42}$ For densitometric analysis, the Gene Tools (Syngene, UK) software was used to compare the protein corona adsorbed on T2LNPs and on nanobeads surface.

\section{Results and discussion}

\section{T2LNPs production by mechanical fragmentation}

Mechanical fragmentation was chosen among several methods to produce true-to-life samples of nanopolystyrene plastics (T2LNPs), mimicking the natural plastic degradation processes caused by rocks and water erosion forces. This fragmentation process was carried out using an ultracentrifugal mill, which allows a rapid polymer fragmentation, with the great advantage of preserving the sample from environmental contaminants and allowing to regulate the process. However, the well-known disadvantage of this method is the generation of local heat, causing thermal degradation of the polymer. For this reason, the samples have been kept in cryogenic conditions during the fragmentation. Before the milling procedure, the plastic items have been embrittled in liquid nitrogen for 30 minutes.

Before the separation and centrifugation steps, the fragmented pieces were suspended in MilliQ water. The proper pre-treatment of MilliQ water represented a crucial step to avoid contaminations. Figure S1a-c in Supporting Information shows AFM images of untreated control water following the same concentration 
steps as T2LNPs where contaminant nanoparticles are detected. The mandatory need to obtain pure preparations of T2LNPs led to a water pre-treatment, with neglecting contaminants, as shown in Figure S1d-f in Supporting Information.

\section{T2LNPs characterization by FT-IR spectroscopy}

Vibrational spectroscopic techniques are recognized as valuable methods for plastics identifications. For example, in plastic recycling plants, vibrational spectroscopy techniques are the basis of automated processes for plastic types sorting, in place of or in combination with manual separation, which is timeconsuming and more prone to errors. ${ }^{43}$ Mid-Infrared transmission spectroscopy is widely used for bulk polymers for both qualitative and quantitative analysis, ${ }^{44}$ and it is also considered an accurate technique to investigate biological materials. ${ }^{45}$ It provides unique fingerprint spectra, using wavelengths ranging from $4000 \mathrm{~cm}^{-1}$ to $400 \mathrm{~cm}^{-1}$. Near-Infrared spectroscopy (NIR), using wavelengths ranging from $780 \mathrm{~nm}$ to 2500 $\mathrm{nm}$, is gaining attention thanks to the development of miniaturized, portable, and real-time NIR spectrometers. ${ }^{46}$ Raman spectroscopy is also used for its greater simplicity in preparing the sample to be analyzed. While wide overtone bands can be obtained with NIR spectroscopy, the Raman spectrum has the advantage of sharper and more well-resolved bands. ${ }^{47}$ However, it has some limits, especially with nonhomogeneous powdered samples and colloidal suspensions. ${ }^{44}$

T2LNPs samples was analyzed by FT-IR spectroscopy operating in transmission mode. Figure 2 shows FT-IR spectra recorded on parental polystyrene macro pieces, fragmented T2LNPs and nanobeads. The spectrum of macro pieces displays characteristic polystyrene absorption bands, namely, peaks at 3083, 3060 and $3027 \mathrm{~cm}^{-1}$, corresponding to aromatic $\mathrm{C}-\mathrm{H}$ stretching vibrations; peaks at $2925 \mathrm{~cm}^{-1}$ and $2852 \mathrm{~cm}^{-1}$, corresponding to the asymmetric and symmetric stretching vibrations of methyl $\left(-\mathrm{CH}_{3}\right)$ and methylene ($\mathrm{CH}_{2}$ ) groups; peak at $1602 \mathrm{~cm}^{-1}$, corresponding to aromatic ring stretching vibrations; and peaks at $1492 \mathrm{~cm}^{-}$ ${ }^{1}$ and $1452 \mathrm{~cm}^{-1}$, corresponding to the asymmetric and symmetric bending vibrations of methyl $\left(-\mathrm{CH}_{3}\right)$ and methylene $\left(-\mathrm{CH}_{2}\right)$ groups and to aromatic ring stretching vibrations. ${ }^{48,49}$ FT-IR analysis revealed the presence of the typical peaks also in fragmented T2LNPs samples, confirming the chemical fingerprint. Due to the low concentration of T2LNPs samples, some absorbance peaks are lost, and the intensity of main adsorption bands is lower compared to bulk material, but it is possible to clearly observe $\mathrm{C}-\mathrm{H}$ characteristic stretches at 3085, 3060 and $3029 \mathrm{~cm}^{-1}$, methyl and methylene stretches at 2920 and $2852 \mathrm{~cm}^{-1}$, aromatic ring stretch at $1602 \mathrm{~cm}^{-1}$ and methyl and methylene bending, together with aromatic ring stretch at 1492 and $1452 \mathrm{~cm}^{-1}$. This behaviour was also confirmed for nanobeads samples. FT-IR analysis confirmed the presence of chemically intact polymer after fragmentation procedure without induced degradations. Mechanical fragmentation coupled with the use of liquid nitrogen is essential to preserve the parental nature of the T2LNPs (see Figure S2 in Supporting Information for an example of polymer degradation after 
a not proper protocol of mechanical fragmentation with a time of embrittlement restricted to 10 minutes and without a continuous flow of liquid nitrogen during the process).

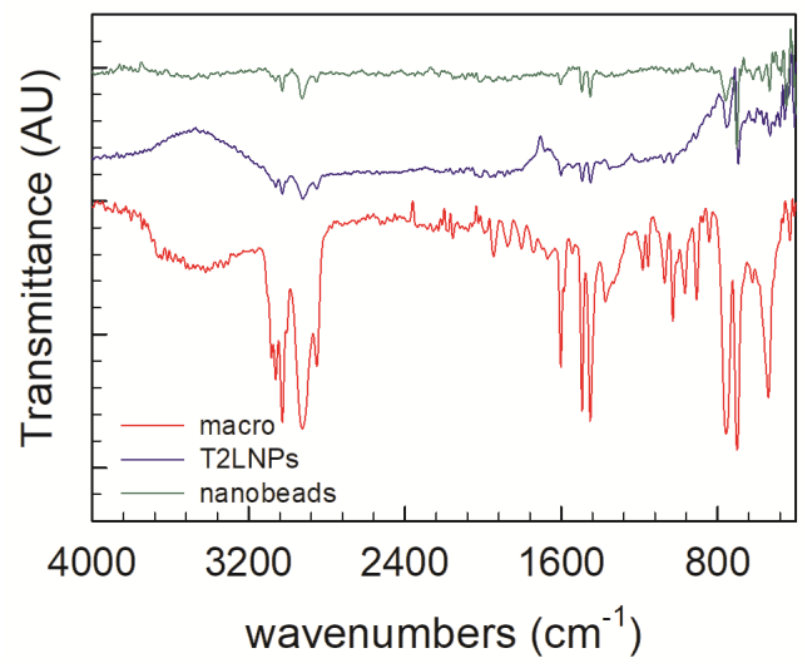

Figure 2. Sample FT-IR spectra, collected in transmission mode, of macro polystyrene macro pieces, true-tolife nanoplastics (T2LNPs) and synthetic nanobeads (nanobeads).

\section{Morphological characterization and size distribution of T2LNPs}

AFM was used to investigate the morphology of single particles and aggregates in the T2LNPs sample. AFM was also employed to foresee the size distribution, taking advantage of non-monodispersed solutions over traditional optical correlation techniques, such as Dynamic Light Scattering (DLS) and Nanoparticle Tracking Analysis (NTA). ${ }^{50}$ It has been demonstrated that sample polydispersity can distort DLS results since the population of big particles in the sample can screen the smaller ones. ${ }^{51}$ On the other hand, NTA tracks the movement of single particles, so it better determines the size even in polydisperse systems. However, many works report that few large particles negatively affect the detection of smaller ones, reducing their quantification up to $80 \% .{ }^{52,53}$ Furthermore, studies on the accuracy of these analytical tools are usually conducted by mixing few populations of well-defined different sized nanoparticles. On the contrary, in the case of true-to-life T2LNPs sample, we expect a large variability in size from few nanometers particles up to large aggregates of hundreds of nanometers; therefore, the accuracy and reliability of such measurements could be further compromised. Lastly, the NTA requires the presence of specialized operators and is strongly influenced by the manual skills of the operator, as well as by the type of instrument, software and calibration used..$^{54}$

Topography AFM images were acquired at different length scales to extrapolate the size distribution and the shapes of the T2LNPs. Figure 3 displays representative images recorded with a scan size set to $25 \mu \mathrm{m}$ (ac), $10 \mu \mathrm{m}(\mathrm{d}-\mathrm{f}), 5 \mu \mathrm{m}(\mathrm{g}-\mathrm{i}), 2 \mu \mathrm{m}(\mathrm{j}-\mathrm{l})$. The most populated fraction of T2LNPs sample is few tens of nanometers in size. The mismatch in z-dimension and diameter observed for some round-shaped particles 
suggests the aggregation of nanoparticles with a resultant tip-sample convolution effect, and/or the presence of elongated particles. ${ }^{33}$ Aggregates of different sizes and shapes are also clearly detected, reaching in some cases a dimension larger than $1 \mu \mathrm{m}$ in diameter.

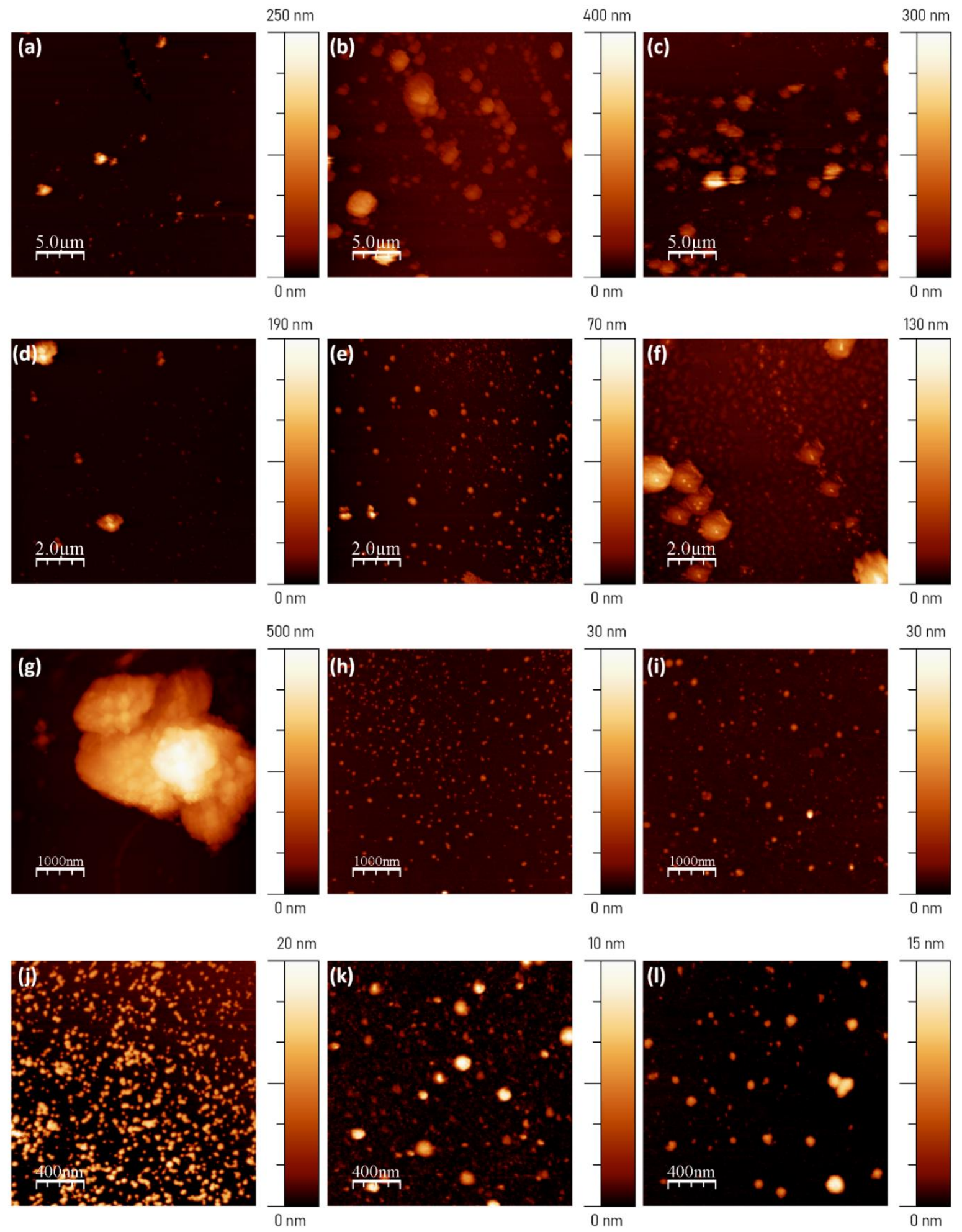

Figure 3. Atomic Force Microscope (AFM) topography images of T2LNPs adsorbed on mica, with scan size of $25 \mu \mathrm{m}(\mathrm{a}-\mathrm{c}), 10 \mu \mathrm{m}(\mathrm{d}-\mathrm{f}), 5 \mu \mathrm{m}(\mathrm{g}-\mathrm{i}), 2 \mu \mathrm{m}(\mathrm{j}-\mathrm{I})$, and scale bars as indicated. Colorimetric scales indicate maximum height for each image. 
Two different T2LNPs samples independently prepared were analyzed, summing up to 20,000 particles giving a strong and statistically representative distribution. Both diameter and z-dimension parameters were measured, considering the presence of in-plane aggregates or elongated particles, as suggested from topography images, and to avoid tip-driven AFM artefacts.

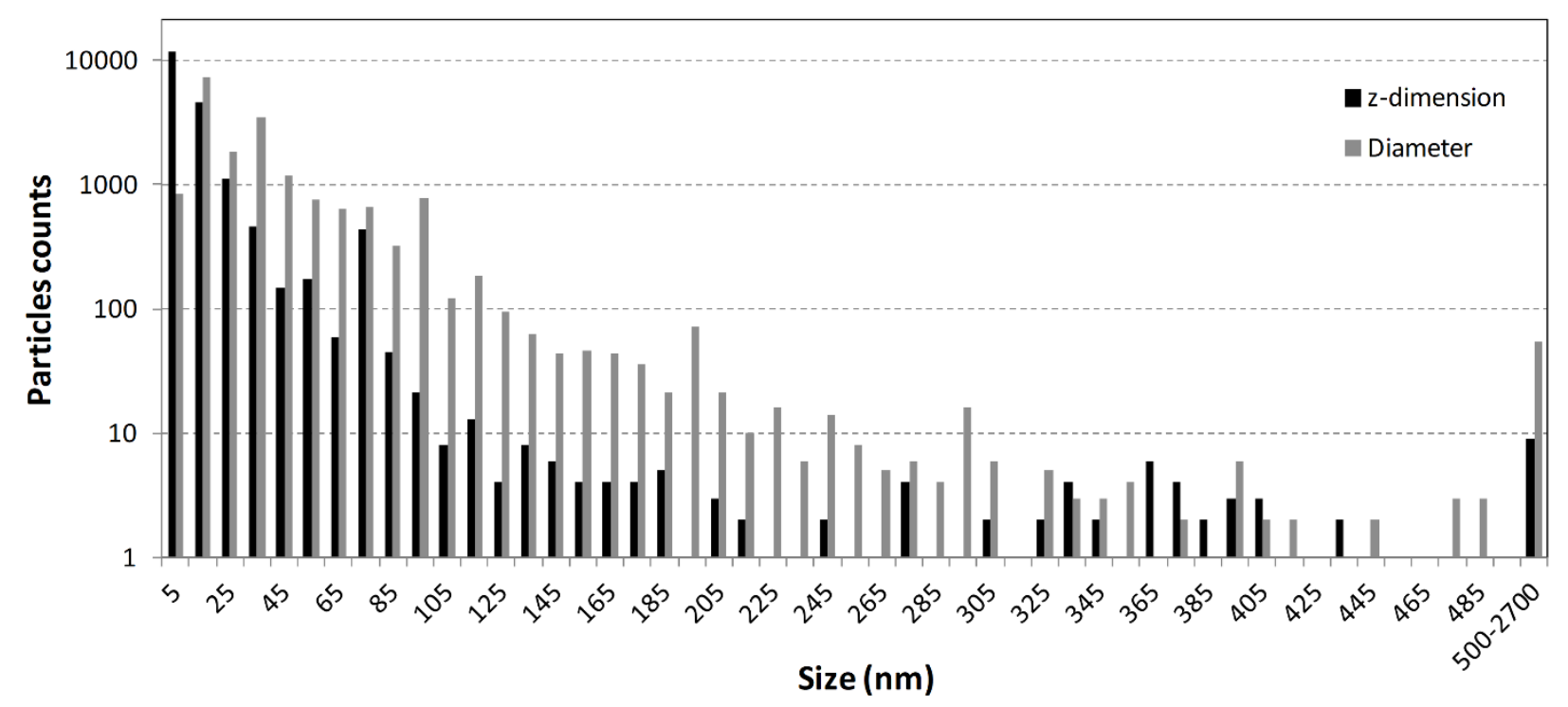

Figure 4. Size distribution obtained from AFM images of two independent preparations of T2LNPs. A grand total of 18,678 objects were analyzed (y axis, log scale).

Figure 4 shows the overall AFM size distribution data of the two analyzed preparations of T2LNPS highlighting the predominance of small particles, below $100 \mathrm{~nm}$ in both $\mathrm{x}-, \mathrm{y}$ - and $\mathrm{z}$ - dimensions, according to topography data. The size distribution shows median values of z-dimension and diameter of $7.5 \mathrm{~nm}$ and $24.0 \mathrm{~nm}$, respectively. The mean values for z-dimension and diameter, more affected by the presence of big aggregates, are $(13.5 \pm 0.2) \mathrm{nm}$ and $(43.0 \pm 0.5) \mathrm{nm}$, respectively.

An inter-samples analysis was performed, by comparing the two different T2LNPs samples. Size distributions and statistical details (Figure S4 and Table S1 in Supporting Information) provides median values of z-dimension of $6.8 \mathrm{~nm}$ for the first sample and $8.0 \mathrm{~nm}$ for the second one. In comparison, median values of diameter are $22.9 \mathrm{~nm}$ and $27.6 \mathrm{~nm}$ for the two samples. Mean values of z-dimension and diameter are $(14.2 \pm 0.2) \mathrm{nm}$ and $(41.3 \pm 0.5) \mathrm{nm}$, respectively, for the first sample, and $(12.9 \pm 0.3) \mathrm{nm}$ and $(44.7 \pm 0.8) \mathrm{nm}$, respectively, for the second sample. These data confirm the repeatability of the T2LNPs production, with a strong predominance of particles with a diameter smaller than $100 \mathrm{~nm}$.

In Figure S3 in Supporting Information a representative AFM topography image of nanobeads is reported, clearly showing a regular particles distribution and a uniform size of about $165 \mathrm{~nm}$ (z-scale). 


\section{Protein corona on T2LNPs and nanobeads}

After determining the morphological diversity between T2LNPs and nanobeads, we investigated their ability to interact with biological environments. Given the fundamental role of the protein corona in defining the final identity of the nanoparticles and their communication interface, ${ }^{34}$ we compared the protein corona adsorbed on T2LNP and nanobead surface after their incubation with human plasma. To investigate this interaction, T2LNPs and nanobeads were incubated with plasma diluted 1:2 in Milli-Q water for $1 \mathrm{~h}$ at $4^{\circ} \mathrm{C}$. After incubation, samples were diluted 1:2 with Milli-Q water, centrifuged and washed as described in the Material and Methods section. A plasma sample, not previously incubated with nanoparticles, was processed in parallel and used as negative control (CTRL-) to check the non-specific signal due to protein interaction with plastic tubes.

The first analysis of the protein corona molecules was performed by FT-IR measurements. The spectra are reported in Figure S5 in Supporting Information and confirmed the presence of the protein film, showing the characteristic adsorption bands ascribed to amide I and amide II bands around $1650 \mathrm{~cm}^{-1}$ and $1540 \mathrm{~cm}^{-}$ ${ }^{1}$, primarily due to the carbonyl stretching vibration and $\mathrm{N}-\mathrm{H}$ bending vibrations $\mathrm{s}^{55}$, respectively. A further morphological check was performed by AFM. The topography images did not show significant modifications of the morphologies, neglecting the contribution of the protein thin film on the T2LNPs surface (Figure S6 Supporting Information). This result was expected since in literature protein corona is described as a thin layer with a thickness of few nanometers, corresponding in some cases to a protein monolayer ${ }^{56,36}$ For this reason, differences in nanoparticles diameter before and after the formation of protein corona is unlikely to be detected.

To visualize differences in the protein composition of the corona adsorbed on T2LNPs and nanobeads, the samples were heated in reducing sample buffer, separated by SDS-PAGE electrophoresis on acrylamide/bisacrylamide gel and stained with Imperial staining (Figure 5). SDS-PAGE allowed to separate proteins based on their molecular weight and the resulting protein bands in the gel were stained using a Coomassie based dye. At first glance, the profile of the protein corona adsorbed on T2LNPs is striking different in comparison with the profile of nanobeads, and both do not mirror the plasmatic total protein pattern (plasma). 


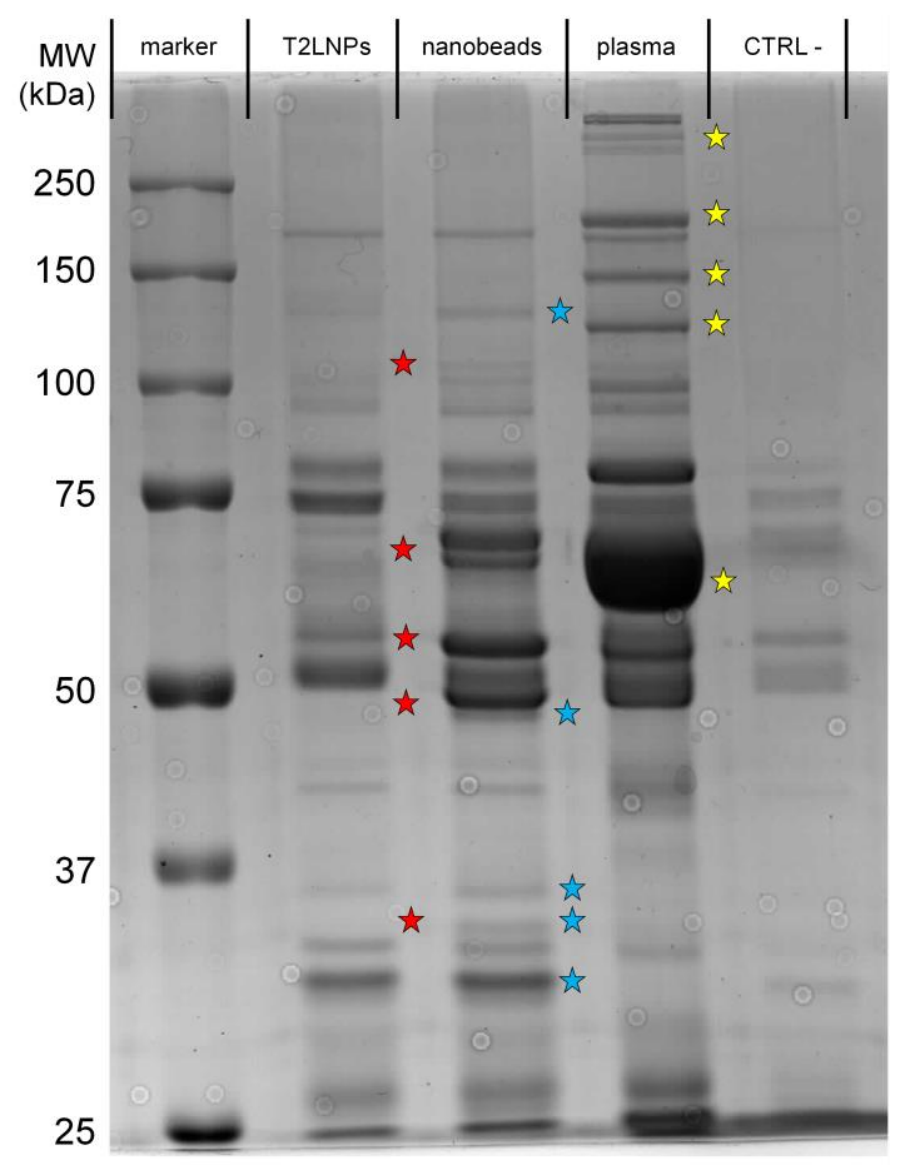

Figure 5. Imperial staining of protein corona absorbed on T2LNPS and nanobeads after human plasma incubation, human plasma (plasma, $20 \mu \mathrm{g}$ ) and human plasma processed as T2LNPs and nanobeads corona formation protocol, but without the nanoparticle incubation (CTRL-). All samples were subjected to SDSPAGE on a $7.5 \%$ acrylamide gel and stained with Imperial staining. Differences in protein patterns are highlighted with stars as described in the text. The image is representative of three independent experiments.

In addition, CTRL- showed lower signal intensity and a profile less rich in bands compared to all the other samples, highlighting the specificity of the signals in T2LNPs and nanobeads lanes.

Moreover, some protein bands that are low concentrated in plasma are selectively enriched in the two protein coronas (blue stars). On the other hand, other protein bands abundant in plasma have less intense signal in the two nanomaterial samples (yellow stars). Albumin is the most representative case. It is the most abundant protein in blood plasma, representing approximately $55 \%$ of total blood proteins. It has a molecular weight of $66.5 \mathrm{kDa}$ and clearly emerges from the SDS-PAGE analysis on plasma. However, in the protein patterns of the two nanomaterial-protein complexes, only a less intense signal can be observed (almost absent in the case of the T2LNPs). These findings are in full agreement with the literature. In fact, although some studies of interaction between albumin and nanobeads show the formation of a protein corona, ${ }^{57,35}$ a different behavior is experienced in testing a complex fluid, such as human plasma. In this 
case, it was shown that the predominant proteins in the corona are mainly the three subunits $\alpha-, \beta$ - and $\gamma^{-}$ of fibrinogen, while albumin represents a small percentage of the total amount of bound proteins..$^{58,59}$ Differences in protein corona profiles of T2LNPs and nanobeads are highlighted in Figure 5 with red stars and can be even better appreciated analyzing the densitometric profile of the two lanes respectively (Figure 6a). The greatest differences in the densitometric profiles can be observed in the range between 150 and $50 \mathrm{kDa}$ where some proteins are selectively adsorbed either on T2LNPs or on nanobeads (Figure 6a). In addition, densitometric profile data elaboration, showing the relative abundances of proteins grouped on the basis of their molecular weight (Figure 6b), highlighted that both the coronas are formed mostly by proteins with a mass $<75 \mathrm{kDa}$, even if the relative abundance of the different molecular weight groups, in respect to the total amount of protein, is different (46.2\% (MW 50-75 kDa), 4.2\% (MW 37-50 $\mathrm{kDa}$ ), 25.4\% (MW < $37 \mathrm{kDa}$ ) and 43.2\% (MW 50-75 kDa), 13.3\% (MW 37-50 kDa), 23.5\% (MW < $37 \mathrm{kDa}$ ) for T2LNPs and nanobeads, respectively) (Figure 6b). This result demonstrates the need of the representative sample to study the nanoplastics-bio interactions on environmental NPs.
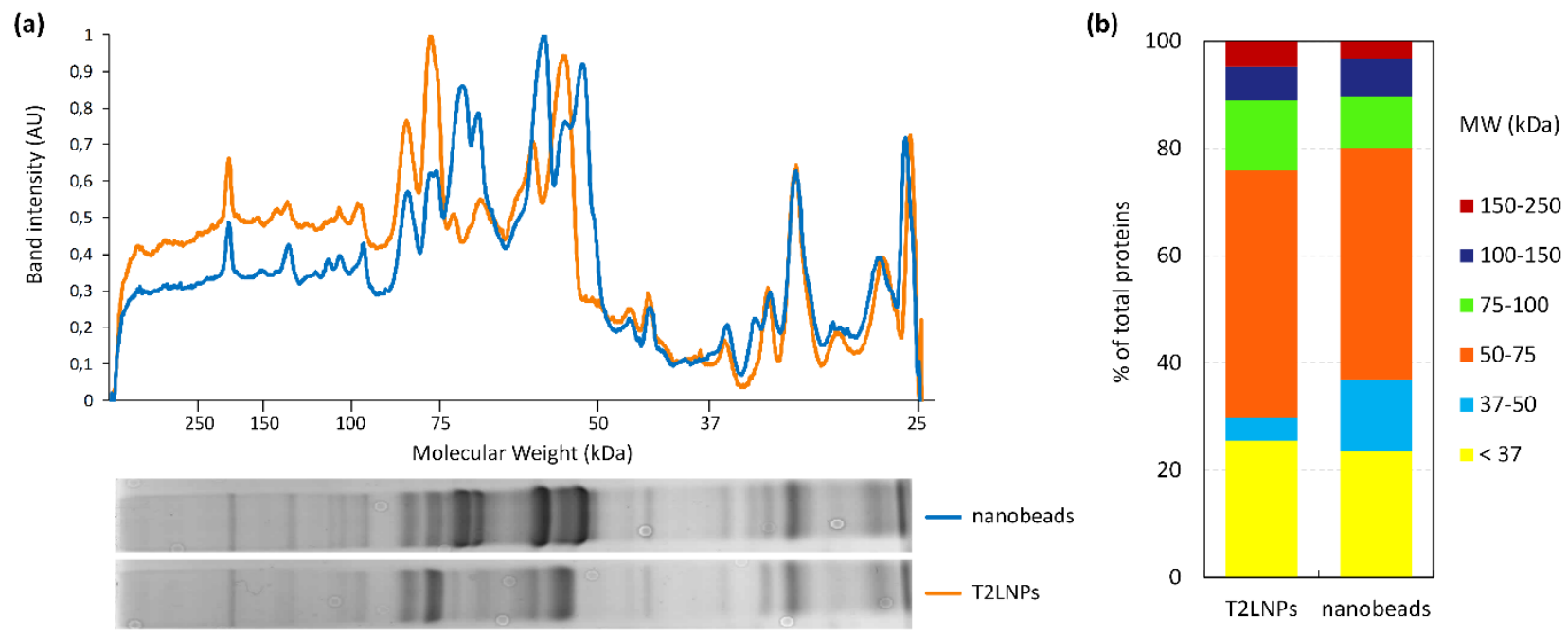

Figure 6. a) Densitometric profiles of the protein corona adsorbed on T2LNPs (orange line) and on nanobeads (blue line). b) Densitometric profile data elaboration shows the relative abundance (\% of total proteins) of the different molecular weight groups (MW), in respect to the total amount of protein for each lane (T2LNPs and nanobeads).

\section{Conclusions}

The number of studies reporting the effects of nanoplastics on biota is growing exponentially, and they are commonly based on the use of synthetic nanobeads as models for nanoplastics. These particles have been of utmost importance to standardize methodologies and begin to unravel the fundamental mechanisms of interaction of nanoplastics with biological systems. There is a strong need to integrate field and laboratory data and test more environmentally relevant conditions. ${ }^{15}$ and to define a standard reference sample that 
mirror the chemical composition and the variety in morphology and size of naturally-occurring nanoplastics, yet keeping reasonably narrow production repeatability and physicochemical characteristics. In this work, we propose a "true-to-life" nanoplastics (T2LNPs) reference sample produced by replicating the breakdown process originating in nature due to mechanical forces by mechanical fragmentation in cryogenic conditions of polystyrene-based daily-life items.

The features recorded in topography images showed spheroidal nanoparticles, with a strong predominance of tiny particles of few tens of nanometers, also combined with aggregates and bigger particles. The richness of the information achieved confirmed AFM as a valuable technique to characterize the size distribution of nanoplastics. The bio-nanointerface was here investigated through the formation of the protein corona from human plasma both on the T2LNPs and nanobeads surface, highlighting different protein profiles for the two nanomaterials. The composition and structure of the protein corona are strictly connected to the chemistry and nanoparticle surface features. There are strong indications that the curvature radius of the nanoparticle is also a key parameter. Fine details of size, shape and bare-surface physicochemical properties can lead to different protein corona ${ }^{60}$ and further characterizations at different levels needs to be performed to define the major contributors to the differences between the protein corona formed on T2LNPs and nanobeads. Nevertheless, the differences detected in the two protein corona profiles confirm the gap between controlled models and the complexity in real-life scenarios, supporting the need to develop true-to-life materials as reasonable models for environmental nanoplastics. Given that the protein corona mediates the interaction with biological systems and the surroundings, different compositions of protein corona strongly influence the potential risks of NPs pollution, for the environment and human health.

In conclusion, the broad heterogeneity in size and shape shown by fragmented T2LNPs gives the nanomaterial a peculiar and different behavior compared to the defined pristine nature of nanobeads, nominating T2LNPs as a more faithful material for naturally-occurring nanoplastics and opening the possibility to new and unexpected results in biological interactions.

\section{Author Contributions}

Conceptualization, S.F.; Formal analysis, C.M.; Funding acquisition, L.E.D., P.B.; Investigation, S.D., R.N., L.P., A.Z; Methodology, S.D., L.P., A.R.; Resources, L.E.D., P.B.; Supervision, S.F., P.B.; Visualization: S.D, L.P, A.R., A.Z.; Writing-original draft, S.D., S.F., P.B.; Writing-review and editing, S.F., P.B., L.P., A.R.

\section{Acknowledgments}

This research was funded by PON "R\&I" 2014-2020: SIRIMAP-SIstemi di Rilevamento dell'Inquinamento MArino da Plastiche e successivo recupero-riciclo (No. ARS01_01183, CUP D86C18000520008) and by National Interuniversity Consortium of Materials Science and Technology (INSTM) through internal funds. 


\section{Bibliography}

(1) UNEP. Marine Plastic Debris and Microplastics - Global Lessons and Research to Inspire Action and Guide Policy Change; 2016.

(2) Geyer, R.; Jambeck, J. R.; Law, K. L. Production, Use, and Fate of All Plastics Ever Made. Sci. Adv. 2017, 3 (7), 25-29. https://doi.org/10.1126/sciadv.1700782.

(3) Patrício Silva, A. L.; Prata, J. C.; Walker, T. R.; Duarte, A. C.; Ouyang, W.; Barcelò, D.; Rocha-Santos, T. Increased Plastic Pollution Due to COVID-19 Pandemic: Challenges and Recommendations. Chemical Engineering Journal. Elsevier B.V. February 1, 2021, p 126683. https://doi.org/10.1016/j.cej.2020.126683.

(4) Andrady, A. L. Microplastics in the Marine Environment. Marine Pollution Bulletin. Elsevier Ltd 2011, pp 1596-1605. https://doi.org/10.1016/j.marpolbul.2011.05.030.

(5) Dawson, A. L.; Kawaguchi, S.; King, C. K.; Townsend, K. A.; King, R.; Huston, W. M.; Bengtson Nash, S. M. Turning Microplastics into Nanoplastics through Digestive Fragmentation by Antarctic Krill. Nat. Commun. 2018, 9 (1), 1-8. https://doi.org/10.1038/s41467-018-03465-9.

(6) Andrady, A. L. The Plastic in Microplastics: A Review. Mar. Pollut. Bull. 2017, 119 (1), 12-22. https://doi.org/10.1016/j.marpolbul.2017.01.082.

(7) Wang, L.; Wu, W. M.; Bolan, N. S.; Tsang, D. C. W.; Li, Y.; Qin, M.; Hou, D. Environmental Fate, Toxicity and Risk Management Strategies of Nanoplastics in the Environment: Current Status and Future Perspectives. J. Hazard. Mater. 2021, 401, 123415. https://doi.org/10.1016/j.jhazmat.2020.123415.

(8) Peng, L.; Fu, D.; Qi, H.; Lan, C. Q.; Yu, H.; Ge, C. Micro- and Nano-Plastics in Marine Environment: Source, Distribution and Threats - A Review. Sci. Total Environ. 2020, 698, 134254. https://doi.org/10.1016/j.scitotenv.2019.134254.

(9) Torres, F. G.; Dioses-Salinas, D. C.; Pizarro-Ortega, C. I.; De-la-Torre, G. E. Sorption of Chemical Contaminants on Degradable and Non-Degradable Microplastics: Recent Progress and Research Trends. Science of the Total Environment. Elsevier B.V. February 25, 2021, p 143875. https://doi.org/10.1016/j.scitotenv.2020.143875.

(10) Banerjee, A.; Shelver, W. L. Micro- and Nanoplastic Induced Cellular Toxicity in Mammals: A Review. Science of the Total Environment. Elsevier B.V. February 10, 2021, p 142518. https://doi.org/10.1016/j.scitotenv.2020.142518.

(11) Liao, Y. liang; Yang, J. yan. Microplastic Serves as a Potential Vector for $\mathrm{Cr}$ in an In-Vitro Human Digestive Model. Sci. Total Environ. 2020, 703, 134805. https://doi.org/10.1016/j.scitotenv.2019.134805.

(12) Cole, M.; Lindeque, P.; Halsband, C.; Galloway, T. S. Microplastics as Contaminants in the Marine 
Environment: A Review. Mar. Pollut. Bull. 2011, 62 (12), 2588-2597.

https://doi.org/10.1016/j.marpolbul.2011.09.025.

(13) Barboza, L. G. A.; Dick Vethaak, A.; Lavorante, B. R. B. O.; Lundebye, A. K.; Guilhermino, L. Marine Microplastic Debris: An Emerging Issue for Food Security, Food Safety and Human Health. Mar. Pollut. Bull. 2018, 133 (January), 336-348. https://doi.org/10.1016/j.marpolbul.2018.05.047.

(14) Hahladakis, J. N.; Velis, C. A.; Weber, R.; lacovidou, E.; Purnell, P. An Overview of Chemical Additives Present in Plastics: Migration, Release, Fate and Environmental Impact during Their Use, Disposal and Recycling. J. Hazard. Mater. 2018, 344, 179-199.

https://doi.org/10.1016/j.jhazmat.2017.10.014.

(15) Oliveira, M.; Almeida, M. The Why and How of Micro(Nano)Plastic Research. TrAC - Trends Anal. Chem. 2019, 114, 196-201. https://doi.org/10.1016/j.trac.2019.02.023.

(16) Nguyen, B.; Claveau-Mallet, D.; Hernandez, L. M.; Xu, E. G.; Farner, J. M.; Tufenkji, N. Separation and Analysis of Microplastics and Nanoplastics in Complex Environmental Samples. Acc. Chem. Res. 2019, 52 (4), 858-866. https://doi.org/10.1021/acs.accounts.8b00602.

(17) Gigault, J.; Halle, A. ter; Baudrimont, M.; Pascal, P. Y.; Gauffre, F.; Phi, T. L.; El Hadri, H.; Grassl, B.; Reynaud, S. Current Opinion: What Is a Nanoplastic? Environ. Pollut. 2018, 235, 1030-1034. https://doi.org/10.1016/j.envpol.2018.01.024.

(18) Wu, J.; Jiang, R.; Lin, W.; Ouyang, G. Effect of Salinity and Humic Acid on the Aggregation and Toxicity of Polystyrene Nanoplastics with Different Functional Groups and Charges. Environ. Pollut. 2019, 245, 836-843. https://doi.org/10.1016/j.envpol.2018.11.055.

(19) Liu, L.; Xu, K.; Zhang, B.; Ye, Y.; Zhang, Q.; Jiang, W. Cellular Internalization and Release of Polystyrene Microplastics and Nanoplastics. Sci. Total Environ. 2021, 779, 146523. https://doi.org/10.1016/j.scitotenv.2021.146523.

(20) Tallec, K.; Blard, O.; González-Fernández, C.; Brotons, G.; Berchel, M.; Soudant, P.; Huvet, A.; PaulPont, I. Surface Functionalization Determines Behavior of Nanoplastic Solutions in Model Aquatic Environments. Chemosphere 2019, 225, 639-646.

https://doi.org/10.1016/j.chemosphere.2019.03.077.

(21) Sun, X.; Chen, B.; Li, Q.; Liu, N.; Xia, B.; Zhu, L.; Qu, K. Toxicities of Polystyrene Nano- and Microplastics toward Marine Bacterium Halomonas Alkaliphila. Sci. Total Environ. 2018, 642, 13781385. https://doi.org/10.1016/j.scitotenv.2018.06.141.

(22) Lin, W.; Jiang, R.; Hu, S.; Xiao, X.; Wu, J.; Wei, S.; Xiong, Y.; Ouyang, G. Investigating the Toxicities of Different Functionalized Polystyrene Nanoplastics on Daphnia Magna. Ecotoxicol. Environ. Saf. 2019, 180 (May), 509-516. https://doi.org/10.1016/j.ecoenv.2019.05.036.

(23) Zhang, W.; Liu, Z.; Tang, S.; Li, D.; Jiang, Q.; Zhang, T. Transcriptional Response Provides Insights into the Effect of Chronic Polystyrene Nanoplastic Exposure on Daphnia Pulex. Chemosphere 2020, 238, 
124563. https://doi.org/10.1016/j.chemosphere.2019.124563.

(24) Guimarães, A. T. B.; Estrela, F. N.; Rodrigues, A. S. de L.; Chagas, T. Q.; Pereira, P. S.; Silva, F. G.; Malafaia, G. Nanopolystyrene Particles at Environmentally Relevant Concentrations Causes Behavioral and Biochemical Changes in Juvenile Grass Carp (Ctenopharyngodon Idella). J. Hazard. Mater. 2021, 403, 123864. https://doi.org/10.1016/j.jhazmat.2020.123864.

(25) Sökmen, T. Ö.; Sulukan, E.; Türkoğlu, M.; Baran, A.; Özkaraca, M.; Ceyhun, S. B. Polystyrene Nanoplastics (20 Nm) Are Able to Bioaccumulate and Cause Oxidative DNA Damages in the Brain Tissue of Zebrafish Embryo (Danio Rerio). Neurotoxicology 2020, 77 (July 2019), 51-59. https://doi.org/10.1016/j.neuro.2019.12.010.

(26) Yu, C. W.; Luk, T. C.; Liao, V. H. C. Long-Term Nanoplastics Exposure Results in Multi and TransGenerational Reproduction Decline Associated with Germline Toxicity and Epigenetic Regulation in Caenorhabditis Elegans. J. Hazard. Mater. 2021, 412, 125173.

https://doi.org/10.1016/j.jhazmat.2021.125173.

(27) Yin, K.; Wang, Y.; Zhao, H.; Wang, D.; Guo, M.; Mu, M.; Liu, Y.; Nie, X.; Li, B.; Li, J.; Xing, M. A Comparative Review of Microplastics and Nanoplastics: Toxicity Hazards on Digestive, Reproductive and Nervous System. Sci. Total Environ. 2021, 774, 145758. https://doi.org/10.1016/j.scitotenv.2021.145758.

(28) Gaylarde, C. C.; Baptista Neto, J. A.; da Fonseca, E. M. Nanoplastics in Aquatic Systems - Are They More Hazardous than Microplastics? Environ. Pollut. 2021, 272. https://doi.org/10.1016/j.envpol.2020.115950.

(29) Magrì, D.; Sánchez-Moreno, P.; Caputo, G.; Gatto, F.; Veronesi, M.; Bardi, G.; Catelani, T.; Guarnieri, D.; Athanassiou, A.; Pompa, P. P.; Fragouli, D. Laser Ablation as a Versatile Tool to Mimic Polyethylene Terephthalate Nanoplastic Pollutants: Characterization and Toxicology Assessment. ACS Nano 2018, 12 (8), 7690-7700. https://doi.org/10.1021/acsnano.8b01331.

(30) Lambert, S.; Wagner, M. Characterisation of Nanoplastics during the Degradation of Polystyrene. Chemosphere 2016, 145, 265-268. https://doi.org/10.1016/j.chemosphere.2015.11.078.

(31) Lambert, S.; Wagner, M. Formation of Microscopic Particles during the Degradation of Different Polymers. Chemosphere 2016, 161, 510-517. https://doi.org/10.1016/j.chemosphere.2016.07.042.

(32) Eitzen, L.; Paul, S.; Braun, U.; Altmann, K.; Jekel, M.; Sebastian, A. The Challenge in Preparing Particle Suspensions for Aquatic Microplastic Research. Environ. Res. 2019, 168 (April 2018), 490-495. https://doi.org/10.1016/j.envres.2018.09.008.

(33) Ekvall, M. T.; Lundqvist, M.; Kelpsiene, E.; Šileikis, E.; Gunnarsson, S. B.; Cedervall, T. Nanoplastics Formed during the Mechanical Breakdown of Daily-Use Polystyrene Products. Nanoscale Adv. 2019, 1 (3), 1055-1061. https://doi.org/10.1039/c8na00210j.

(34) Liu, N.; Tang, M.; Ding, J. The Interaction between Nanoparticles-Protein Corona Complex and Cells 
and Its Toxic Effect on Cells. Chemosphere 2020, 245, 125624.

https://doi.org/10.1016/j.chemosphere.2019.125624.

(35) Gopinath, P. M.; Saranya, V.; Vijayakumar, S.; Mythili Meera, M.; Ruprekha, S.; Kunal, R.; Pranay, A.; Thomas, J.; Mukherjee, A.; Chandrasekaran, N. Assessment on Interactive Prospectives of Nanoplastics with Plasma Proteins and the Toxicological Impacts of Virgin, Coronated and Environmentally Released-Nanoplastics. Sci. Rep. 2019, 9 (1), 1-15. https://doi.org/10.1038/s41598019-45139-6.

(36) Kihara, S.; Ghosh, S.; McDougall, D. R.; Whitten, A. E.; Mata, J. P.; Köper, I.; McGillivray, D. J. Structure of Soft and Hard Protein Corona around Polystyrene Nanoplastics-Particle Size and Protein Types. Biointerphases 2020, 15 (5), 051002. https://doi.org/10.1116/6.0000404. Tan, Y.; Zhu, X.; Wu, D.; Song, E.; Song, Y. Compromised Autophagic Effect of Polystyrene Nanoplastics Mediated by Protein Corona Was Recovered after Lysosomal Degradation of Corona. Environ. Sci. Technol. 2020, 54 (18), 11485-11493. https://doi.org/10.1021/acs.est.0c04097.

(38) Fadare, O. O.; Wan, B.; Liu, K.; Yang, Y.; Zhao, L.; Guo, L. H. Eco-Corona vs Protein Corona: Effects of Humic Substances on Corona Formation and Nanoplastic Particle Toxicity in Daphnia Magna. Environ. Sci. Technol. 2020, 54 (13), 8001-8009. https://doi.org/10.1021/acs.est.0c00615.

(39) Dong, Z.; Hou, Y.; Han, W.; Liu, M.; Wang, J.; Qiu, Y. Protein Corona-Mediated Transport of Nanoplastics in Seawater-Saturated Porous Media. Water Res. 2020, 182, 115978. https://doi.org/10.1016/j.watres.2020.115978.

(40) Horcas, I.; Fernández, R.; Gómez-Rodríguez, J. M.; Colchero, J.; Gómez-Herrero, J.; Baro, A. M. WSXM: A Software for Scanning Probe Microscopy and a Tool for Nanotechnology. Rev. Sci. Instrum. 2007, 78 (1), 013705. https://doi.org/10.1063/1.2432410.

(41) Grossi, I.; Radeghieri, A.; Paolini, L.; Porrini, V.; Pilotto, A.; Padovani, A.; Marengoni, A.; Barbon, A.; Bellucci, A.; Pizzi, M.; Salvi, A.; de Petro, G. MicroRNA-34a-5p Expression in the Plasma and in Its Extracellular Vesicle Fractions in Subjects with Parkinson's Disease: An Exploratory Study. Int. J. Mol. Med. 2021, 47 (2), 533-546. https://doi.org/10.3892/ijmm.2020.4806.

(42) Alvisi, G.; Paolini, L.; Contarini, A.; Zambarda, C.; Antonio, V. Di; Colosini, A.; Mercandelli, N.; Timmoneri, M.; Palù, G.; Caimi, L.; Ricotta, D.; Radeghieri, A. Intersectin Goes Nuclear: Secret Life of an Endocytic Protein. Biochem. J. 2018, 475 (8), 1455-1472. https://doi.org/10.1042/BCJ20170897.

(43) Gundupalli, S. P.; Hait, S.; Thakur, A. A Review on Automated Sorting of Source-Separated Municipal Solid Waste for Recycling. Waste Management. Elsevier Ltd February 1, 2017, pp 56-74. https://doi.org/10.1016/j.wasman.2016.09.015.

(44) Chalmers, J. M.; Everall, N. J. Qualitative and Quantitative Analysis of Plastics, Polymers and Rubbers by Vibrational Spectroscopy. In Handbook of Vibrational Spectroscopy; Everall, N. J., Ed.; John Wiley \& Sons, Ltd: Chichester, UK, 2007. https://doi.org/10.1002/9780470027325.s6101.pub2. 
(45) Paolini, L.; Federici, S.; Consoli, G.; Arceri, D.; Radeghieri, A.; Alessandri, I.; Bergese, P. FourierTransform Infrared (FT-IR) Spectroscopy Fingerprints Subpopulations of Extracellular Vesicles of Different Sizes and Cellular Origin. J. Extracell. Vesicles 2020, 9 (1), 1741174. https://doi.org/10.1080/20013078.2020.1741174.

(46) Rani, M.; Marchesi, C.; Federici, S.; Rovelli, G.; Alessandri, I.; Vassalini, I.; Ducoli, S.; Borgese, L.; Zacco, A.; Bilo, F.; Bontempi, E.; Depero, L. E. Miniaturized Near-Infrared (MicroNIR) Spectrometer in Plastic Waste Sorting. Materials (Basel). 2019, 12 (17), 2740. https://doi.org/10.3390/ma12172740.

(47) Allen, V.; Kalivas, J. H.; Rodriguez, R. G. Post-Consumer Plastic Identification Using Raman Spectroscopy. Appl. Spectrosc. 1999, 53 (6), 672-681. https://doi.org/10.1366/0003702991947324.

(48) Coates, J. Interpretation of Infrared Spectra, A Practical Approach. In Encyclopedia of Analytical Chemistry; John Wiley \& Sons, Ltd: Chichester, UK, 2006.

https://doi.org/10.1002/9780470027318.a5606.

(49) Zolotarev, V. M. Comparison of Polystyrene IR Spectra Obtained by the T, R, ATR, and DR Methods. Opt. Spectrosc. (English Transl. Opt. i Spektrosk. 2017, 122 (5), 749-756. https://doi.org/10.1134/\$0030400X1705023X.

(50) Hoo, C. M.; Starostin, N.; West, P.; Mecartney, M. L. A Comparison of Atomic Force Microscopy (AFM) and Dynamic Light Scattering (DLS) Methods to Characterize Nanoparticle Size Distributions. J. Nanoparticle Res. 2008, 10 (SUPPL. 1), 89-96. https://doi.org/10.1007/s11051-008-9435-7.

(51) Tomaszewska, E.; Soliwoda, K.; Kadziola, K.; Tkacz-Szczesna, B.; Celichowski, G.; Cichomski, M.; Szmaja, W.; Grobelny, J. Detection Limits of DLS and UV-Vis Spectroscopy in Characterization of Polydisperse Nanoparticles Colloids. J. Nanomater. 2013, 2013. https://doi.org/10.1155/2013/313081.

(52) Gardiner, C.; Ferreira, Y. J.; Dragovic, R. A.; Redman, C. W. G.; Sargent, I. L. Extracellular Vesicle Sizing and Enumeration by Nanoparticle Tracking Analysis. J. Extracell. Vesicles 2013, 2 (1), 19671. https://doi.org/10.3402/jev.v2i0.19671.

(53) Montis, C.; Zendrini, A.; Valle, F.; Busatto, S.; Paolini, L.; Radeghieri, A.; Salvatore, A.; Berti, D.; Bergese, P. Size Distribution of Extracellular Vesicles by Optical Correlation Techniques. Colloids Surfaces B Biointerfaces 2017, 158, 331-338. https://doi.org/10.1016/j.colsurfb.2017.06.047.

(54) Vestad, B.; Llorente, A.; Neurauter, A.; Phuyal, S.; Kierulf, B.; Kierulf, P.; Skotland, T.; Sandvig, K.; Haug, K. B. F.; Øvsteb $\varnothing$, R. Size and Concentration Analyses of Extracellular Vesicles by Nanoparticle Tracking Analysis: A Variation Study. J. Extracell. Vesicles 2017, 6 (1), 1344087. https://doi.org/10.1080/20013078.2017.1344087.

(55) Barth, A. Infrared Spectroscopy of Proteins. Biochimica et Biophysica Acta - Bioenergetics. Elsevier September 1, 2007, pp 1073-1101. https://doi.org/10.1016/j.bbabio.2007.06.004.

(56) Casals, E.; Pfaller, T.; Duschl, A.; Oostingh, G. J.; Puntes, V. Time Evolution of the Nanoparticle 
Protein Corona. ACS Nano 2010, 4 (7), 3623-3632. https://doi.org/10.1021/nn901372t.

(57) Kihara, S.; Van Der Heijden, N. J.; Seal, C. K.; Mata, J. P.; Whitten, A. E.; Köper, I.; McGillivray, D. J. Soft and Hard Interactions between Polystyrene Nanoplastics and Human Serum Albumin Protein Corona. Bioconjug. Chem. 2019, 30 (4), 1067-1076.

https://doi.org/10.1021/acs.bioconjchem.9b00015.

(58) Kelly, P. M.; Åberg, C.; Polo, E.; O’Connell, A.; Cookman, J.; Fallon, J.; Krpetić, Ž.; Dawson, K. A. Mapping Protein Binding Sites on the Biomolecular Corona of Nanoparticles. Nat. Nanotechnol. 2015, 10 (5), 472-479. https://doi.org/10.1038/nnano.2015.47.

(59) Monopoli, M. P.; Walczyk, D.; Campbell, A.; Elia, G.; Lynch, I.; Baldelli Bombelli, F.; Dawson, K. A. Physical-Chemical Aspects of Protein Corona: Relevance to in Vitro and in Vivo Biological Impacts of Nanoparticles. J. Am. Chem. Soc. 2011, 133 (8), 2525-2534. https://doi.org/10.1021/ja107583h.

(60) Monopoli, M. P.; Åberg, C.; Salvati, A.; Dawson, K. A. Biomolecular Coronas Provide the Biological Identity of Nanosized Materials. Nat. Nanotechnol. 2012, 7 (12), 779-786.

https://doi.org/10.1038/nnano.2012.207.

TOC

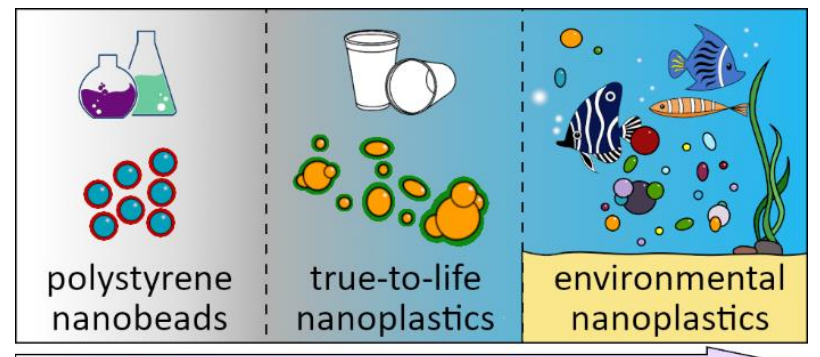

Lab model

Real world 